\title{
Regulierungskrise im Telekommunikationssektor in der BRD
}

Im Folgenden soll von jenen Tendenzen zu einer umfassenden »Entstaatlichung « des Telekommunikationswesens die Rede sein, die in anderen kapitalistischen Industrieländern unter dem Etikett »Deregulierung « für Schlagzeilen sorgen - so die Entflechtung der Fernmeldemonopolgesellschaft AT \& T in den USA, die Privatisierung der britischen Fernmeldebehörde »British Telecom « oder die Teilprivatisierung von »Nippon Telephone and Telegraph« in Japan. Besonders in den USA und Großbritannien sind hier unter den Vorzeichen neo-konservativer Marktideologie Veränderungen in den ökonomisch-politischen Rahmenbedingungen der Entwicklung der Telekommunikation geschaffen worden, die nicht nur zu enormen Arbeitsplatzverlusten in den traditionell gesicherten Beschäftigungsverhältnissen der "communication workers « geführt haben, sondern auch gewerkschaftliche Einflußmöglichkeiten auf die übergreifende Technikentwicklung im Bereich der Informations- und Kommunikations-(IUK-) Netze nahezu gänzlich beseitigen (vgl. z.B. Kohl 1982, Batstone u.a. 1984, ETUI 1984).

Im Gegensatz dazu scheint die Modernisierungspolitik der dominanten Gruppen des Industriekapitals und des Staates in der BRD auf eine recht behutsame Modifizierung der nationalen Konkurrenzbedingungen in der Telekommunikationsbranche hinauszulaufen. Bis heute bildet die Stabilität des Fernmeldemonopols der Deutschen Bundespost den Rahmen für die Existenz solider sozialpartnerschaftlicher Beziehungen zwischen Kapital, Staat und Gewerkschaften im bundesdeutschen Telekommunikationssektor.

Wie ist es zu erklären, daß die Umbrüche auf dem Weltmarkt der Telekommunikation die bestehenden Formen und Mechanismen der Staatsintervention im Telekommunikationssektor der BRD bisher kaum berührt haben, obwohl die westdeutsche Telekommunikationsindustrie als einer der größten Exporteure auf dem Weltmarkt ähnlichen internationalen Anpassungszwängen wie etwa das US-amerikanische oder das japanische Kapital unterliegt? Zu fragen ist, welche spezifischen ökonomischen Bedingungen die relative Stabilität der politischen Regulierungsmechanismen im Telekommunikationssektor der BRD ermöglichen, ob und in welcher Form sich weltmarktvermittelte "Entstaatlichungstendenzen « auch in der $\mathrm{BRD}$ durchsetzen und welche Folgen und Auswirkungen dies für die betroffenen Lohnabhängigen und die sie repräsentierenden Gewerkschaften haben kann. Die Beantwortung dieser Frage erfordert eine genauere Untersuchung der Veränderungen in den Weltmarktstrategien der betreffenden multinationalen Konzerne in der BRD.

\section{Deregulierung und Weltmarktkonkurrenz in der Telekommunikationsbranche}

Versuchen wir zunächst, einige zentrale ökonomische Implikatoren der Regulierungskrise im Telekommunikationswesen der kapitalistischen Industrieländer herauszuarbeiten. Zu Recht bezeichnet Junne (1983) die Entregulierung von Telekommunikationsmärkten als 
eines der wichtigsten Felder des »strukturpolitischen Wettlaufs « zwischen den entwickelten kapitalistischen Nationen in den sog. Hochtechnologieindustrien. Betont werden muß, daß die Umbrüche in den staatlichen Regulierungspraktiken im Telekommunikationsbereich auf strukturelle Ursachen in den Akkumulations- und Konkurrenzverhältnissen des Kapitals zurückzuführen sind. Wir haben es mit einer »Formkrise (vgl. Altvater 1983) von Staatsintervention zu tun, in welcher die in der »fordistischen «Epoche des Kapitalismus dominierenden Formen und Mechanismen staatlicher Infrastruktur unter dem Druck veränderter Akkumulationsverhältnisse zur Disposition stehen.

Im Kalkül der zahlreichen konservativen Verfechter von Deregulierungsmaßnahmen erscheint die Aufhebung der Monopolstellung der nationalen Fernmeldegesellschaften als das Patentrezept, mit dem sowohl die gesamtwirtschaftlichen Rationalisierungswirkungen neuer Kommunikationstechniken durchgesetzt, als auch der Konkurrenzdruck zwischen den Produzenten von Telekommunikationseinrichtungen zur Beschleunigung des technischen Fortschritts intensiviert werden können. Kernargument solcher »Deregulierungstheorien « (vgl. z.B. Kaufer 1981) ist die These vom Ende des »natürlichen Monopols« in der Nachrichtenübermittlung. In der bürgerlichen Wettbewerbstheorie wird das Fernmeldewesen traditionell als ein »wettbewerblicher Ausnahmebereich « angesehen - als ein Markt, in dem »die unternehmerische Kostendegression (economies of scales) in Relation zur gegebenen Marktgröße so wichtig ist, daß im Wettbewerb auf Dauer nur ein Unternehmen überleben würde. Das natürliche Monopol ist somit dadurch gekennzeichnet, daß die im Markt nachgefragte Menge von einem einzigen Anbieter zu niedrigeren Kosten produziert werden kann als von jeder größeren Zahl von Unternehmen. Bei einer derartigen Marktkonzentration würde die Zulassung von Wettbewerb zu einer Vergeudung von Ressourcen führen« (Monopolkommission 1981, S. 25). Die Größenvorteile beim Betrieb von Fernmeldenetzen beruhen im wesentlichen auf technologischen Faktoren, d.h. vor allem auf Kostenvorteilen durch den Netzverbund, der eine langfristige Investitionsplanung ermöglicht bzw. durch »Vermeidung von Doppelinvestitionen« eine rentable Auslastung und Amortisation dieser Investitionen gestattet (ebd.).

Nach Auffassung neo-liberaler Ökonomen ist das »natürliche Monopol« beim Betrieb von Fernmeldezentren durch die Entwicklung der Kommunikationstechnologie heute überholt, weil die enorme Leistungsfähigkeit vollelektronischer Vermittlungs- und Übertragungsysteme einen rentablen Betrieb auch kleinerer und spezialisierter Kommunikationsnetze zuläßt (vgl. z.B. Mestmäcker 1980, Wiley 1980, Knieps 1983).

Dieser Entwicklungstrend verweist darauf, daß das Monopol des Staates im Nachrichtenwesen in der Ökonomie des Kapitaliusmus alles andere als eine »natürliche« Angelegenheit ist. Das staatliche Fernmeldemonopol stellt vielmehr die einer bestimmten Stufe der Produktivkraftentwicklung des Kapitals adäquate Form zur Regulierung der gesamtwirtschaftlichen Infrastrukturaufgabe Nachrichtenübermittlung dar.

»Mit der stofflichen Veränderung des Produktionsprozesses«, so haben z.B. Armanski u.a. zutreffend festgestellt, »ändern sich auch die allgemeinen Produktionsbedingungen. Mit der Revolutionierung und Ausdehnung der Produktion erfolgt ebenso die Notwendigkeit der beständigen Ausweitung und Umwälzung der allgemeinen Produktionsbedingungen (z.B. Kommunikations- und Transportwesen). Umfang und Veränderungsgeschwindigkeit sowie der Grad der Wahrnehmung und Erstellung der allgemeinen Produktionsbedingungen durch den Staat bilden zugleich ein Moment der Größenbewegung staatlicher Lohnarbeit» (Armanski u.a. 1976, S. 17). 
Welches sind nun die Faktoren, die der heute anstehenden Neubestimmung der Grenzen der Staatstätigkeit im Kommunikationswesen den Charakter eines krisenhaft zugespitzten "Strukturzwanges« verleihen?

Die ökonomischen Ursachen dieser Entwicklung sind in den veränderten Akkumulationsund Konkurrenzverhältnissen des Kapitals zu suchen. Der ökonomische "Strukturbruch" (vgl. Altvater u.a. 1983) in der Telekommunikationsindustrie ist als ein komplexer Neustrukturierungsprozeßkapitalistischer Produktions - und Marktökonomie in der Branche zu charakterisieren (zu den Begriffen Produktions- und Marktökonomie vgl. Sohn-Rethel 1974 sowie an diesen anknüpfend Benz-Overhage u.a. 1982).

Von der Marktseite her ist dieser Prozeß dadurch bestimmt, daß die enorme Produktiventfaltung in der Nachrichtenübertragung durch Mikroelektronik, Digitalisierung und optoelektronische Kabeltechnologie die zu einem rentablen Betrieb erforderlichen Mindestgrößen von Kommunikationsnetzen so weit reduziert hat, daß das Angebot von Telekommunikationsnetzen und -diensten heute zu einer ergiebigen Quelle privatunternehmerischer Gewinnmaximierung geworden ist. Zwar existieren von seiten der etablierten Wirtschaftswissenschaft keine präzisen Rentabilitätsberechnungen über das genaue Ausmaß von economies of scalesEffekten in Fernmeldenetzen (vgl. OECD 1983, S. 99 ff.; Knieps 1983), aber die zunehmende Zahl gewinnträchtiger Unternehmen im Bereich privater Telekommunikationsnetze und das Vordringen multinationaler EDV-Konzerne in den Sektor der Telekommunikationsdienstleistungen sagt hier mehr über die veränderten Realitäten aus, als jede statistische Rentabilitätsschätzung.

Größeres Gewicht noch kommt den verschiedenen nationalen Deregulierungspolitiken in ihrer Funktion als Instrument zur Neustrukturierung der Absatzmärkte für die Herstellerkonzerne von Telekommunikationseinrichtungen zu. So hat die Deregulierung der Fernmeldemonopole in den USA, Großbritannien und Japan zur Folge, daß die Telekommunikationsmärkte dieser Länder nunmehr auch ausländischen IUK-Herstellern zugänglich werden. Das Zurückdrängen der auf die Interessen der nationalen Fernmeldekonzerne ausgerichtete Nachfragemacht der staatlichen oder staatlich regulierten Fernmeldemonopole beinhaltet tendenziell die Realisierung eines effektiven Preiswettbewerbes der multinationalen Konzerne der Branche und damit eine weitgehende Homogenisierung des Weltmarktes der Telekommunikationstechnik (vgl. OECD 1933, S. 45 und S. 87).

Die Notwendigkeit der Internationalisierung der Telekommunikationsmärkte erwächst aus der Internationalisierung der Produktionsstrategien des Kapitals und steht in unmittelbarem Zusammenhang mit den veränderten produktionsökonomischen Normen in der Telekommunikationsindustrie. Zwei zentrale Momente dieses Prozesses seien hier benannt:

Zum ersten hat das Zusammenwachsen von Datenverarbeitungs- und Nachricbtentechnik in digitalisierten Telekommunikationssystemen zur Folge, daß auch die Konkurrenzverhältnisse des Branchenkapitals einer umfassenden Neustrukturierung unterliegen. Die Durchsetzung der EDV-Produkttechnologie in der Telekommunikation macht die Kapitale der EDVBranche zu unmittelbaren Konkurrenten der traditionellen Fernmeldekonzerne.

Zum zweiten geht die Integration der Fernmeldeindustrie in den »informationstechnischen Branchenkomplex (vgl. Ernst 1984) einher mit tiefgreifenden Veränderungen an der Produktionsbasis des Kapitals. Die traditionell auf elektromechanische Produkttechnologien ausgerichtete Herstellung von Fernmeldesystemen wird den produktionsökonomischen Normen der EDV-Industrie unterstellt. Die Restrukturierung der Produktionsbasis des Branchenkapitals erfordert eine enorme Steigerung der Kapitalintensität, bedingt durch die prohi- 
bitiv hohen FuE-Aufwendungen und die überproportionalen Fixkostenanteile in den hochautomatisierten Produktionsprozessen für elektronische Vermittlungs- und Übertragungssysteme (eine detaillierte Analyse der produktionsökonomischen Veränderungen in der Fernmeldeindustrie liefern Benz-Overhage u.a. 1982).

Die neue Stufenleiter der economies of scales in der Herstellung von Telekommunikationseinrichtungen steigert die zur Verwertung der konstanten Kapitalvorschüsse notwendigen Absatzmengen in Größenordnungen, die auf den fragmentierten nationalen Märkten der Industrieländer nicht mehr realisierbar sind. In einer Studie des Ifo-Instituts für Wirtschaftberechnung wird geschätzt, daß in der Produktion öffentlicher digitaler Vermittlungssysteme »bereits eine Veränderung des Weltmarktanteils von 5 auf $3 \%$ die Amortisationsdauer der FuE-Aufwendungen um $60 \%$ erhöht «(Ifo 1983, S. 65). Anzumerken ist, daß »im Jahre 1978 dieser Prozentsatz von nur vier Firmen weltweit erreicht wurde. ... Daraus kann gefolgert werden, daß insbesondere in Westeuropa die Erträge auf den nationalen Märkten immer weniger ausreichen, um die Investitionen in neue Nachrichtentechnologien tragen zu können« (ebd.).

Strukturelle Überproduktionstendenzen und der verschärfte internationale Verdrängungswettbewerb bilden heute das Szenarium einer rasanten Internationalisierung der "Produktionsketten« (vgl. Palloix 1979) in der Telekommunikationsindustrie.

Die Internationalisierung der kommunikationstechnischen Produktion vollzieht sich im Kontext einer seit Anfang der achziger Jahre in Gang gekommenen Welle der Kapitalzentralisierung auf Weltmarktebene. Triebfeder dieses globalen Neustrukturierungsprozesses ist der für die multinationalen Konzerne der Branche bestehende Zwang, die zum Aufbau kohärenter internationaler Produktionsketten notwendigen Produktions- und Technologiepotentiale sowie entsprechende Absatzmärkte zu gewinnen.

Ein Knotenpunkt dieser internationalen Zentralisierungsbewegung des Kapitals ist die 1984 in Kraft getretene Entflechtung des US-amerikanischen AT \& T-Konzerns, der nunmehr mit dem erklärten Ziel, die Dominanzstellung von IBM in der IuK-Branche zu brechen, zu einem der wichtigsten Akteure der Weltmarktkonkurrenz geworden ist. Charakteristisch für den gegenwärtigen Internationalisierungsprozeß in der Telekommunikationsindustrie sind etwa die spektakulären Beteiligungen von AT \& T an Olivetti und dem Telekommunikationsbereich von Philips.

Nicht ohne Grund wird in diesem Zusammenhang z.B. in einer Studie des Europäischen Gewerkschaftsinstituts davor gewarnt, daß nach Teilen der Computer- und Halbleiterproduktion in Zukunft auch die kommunikationstechnische Industrie in europäische Ländern zu reinen "point of sale assembly operations« von US-Konzernen verwandelt werden könnte und damit der westeuropäischen Industrie die Kontrolle über strategisch entscheidende Technologiepotentiale entzogen würde (vgl. ETUI 1984, S. 40 ff.).

\section{Fernmeldemonopol und Exportstrategien des Elektrokapitals in der BRD}

Wie ist es nun zu erklären, daß der ökonomische Strukturbruch im Telekommunikationssektor in der Bundesrepublik bislang nicht zu einer weiterreichenden Deregulierung des Fernmeldemonopols geführt hat?

Eine nähere Betrachtung der Akkumulations- und Reproduktionsstrukturen des westdeutschen IuK-Kapitals gibt Aufschluß zur Beantwortung dieser Frage. Es erweist sich, daß die 
von den elektrotechnischen Universalkonzernen Siemens, Bosch und AEG dominierte bundesdeutsche Telekommunikationsindustrie in besonderem Maße von der Produktionsund Technologieentwicklung im Kernbereich der traditionellen Fernmeldeproduktion abhängig ist.

Die Ausgangsbasis des westdeutschen Kapitals in dem gegenwärtigen Neustrukturierungsprozeß von Kapitalakkumulation und Branchenkonkurrenz im Zuge der Verschmelzung von EDV- Fernmelde- und Büromaschinenindustrie läßt sich kurz folgendermaßen charakterisieren: Während die Konzerne der bundesdeutschen Telekommunikationsbranche über eine technologisch hochentwickelte und exportstarke Fernmeldeproduktion verfügen und die Büromaschinenindustrie trotz schwerer Strukturkrisen der führenden Unternehmen (Triumph-Adler und Olympia) nach wie vor eine erstrangige Stellung am Weltmarkt einnehmen kann, ist die internationale Konkurrenzposition des BRD-Kapitals im engeren, strategisch entscheidenden Bereich der EDV-Produktion, d.h. bei Rechnersystemen und Halbleitern vergleichsweise schwach (vgl. z.B. Ifo 1983, BMFT 1984). Hier kann nur der relativ kleine Nixdorf-Konzern eine rasch expandierende internationale Marktstellung vorweisen, während Siemens seine Stellung als drittgrößter Computerhersteller in Westeuropa (vgl. FT v. 4.7.1984) in beträchtlichem Maße seiner Vorzugsstellung bei öffentlichen EDVFörderungsprogrammen in der BRD verdankt (vgl. die Zeit v. 23.3.85). Das nach wie vor relative hohe Exportvolumen der BRD in der EDV-Technik basiert weniger auf den Exportfolgen westdeutscher Hersteller, sondern »im wesentlichen auf den Aktivitäten der hier ansässigen Tochtergesellschaften ausländischer Konzerne (allen voran IBM)« (Ifo 1983, S. 86). Mit einem geschätzten Marktanteil von $36 \%$ (im Gesamtbereich der EDV-Produktion, 1982; vgl. ebd.) ist IBM der dominierende Konzern der EDV-Branche in der BRD. Die IBMDeutschland GmbH ist mit einem Jahresumsatz von über $10 \mathrm{Mrd}$. DM (vgl. Geschäftsbericht 1983) nicht nur die größte Auslandstochtergesellschaft des US-Multis (vgl. FAZ v. 28.9. 1985), sondern auch ein zentrales Glied in den internationalen Produktionsketten dieses Konzerns.

Angesichts ihrer relativen Schwächeposition in der EDV-Technik sind die westdeutschen IuKKonzerne darauf angewiesen, bei der Zusammenfassung der Produktionspotentiale innerhalb des informationstechnischen Branchenkomplexes eine Strategie der »Rückwärtsintegration « (vgl. OECD 1983, Gizycki/Schubert 1984, ETUI 1984) zu verfolgen; die technologische Spitzenstellung und die internationalen Konkurrenzvorsprünge in der Fernmeldeproduktion sind die entscheidenden ökonomischen Aktivposten des BRD-Kapitals bei der »Vernetzung « der Produktionsstrategien im Telekommunikationsbereich. Immerhin ist die bundesdeutsche Fernmeldeindustrie einer der größten Exporteure von nachrichtentechnischen Anlagen auf dem Weltmarkt - nach eigener Darstellung sogar der größte (vgl. OECD 1983 sowie ZVEI 1983) ${ }^{1}$.

In Anbetracht der überproportionalen Dominanz des fernmeldetechnischen Sektors im Produktions- und Reproduktionszusammenhang des IuK-technischen Kapitals der BRD liegt es auf der Hand, daß der Konkurrenzverlauf im traditionellen Marktbereich der Nachrichtentechnik eine, wenn nicht die entscheidende Determinante der internationalen Wettbewerbsfähigkeit der westdeutschen IuK-Konzerne ist.

Der ökonomische und technische Entwicklungsrhythmus der Fernmeldeindustrie wird in der BRD - wie auch in anderen Industrieländern - traditionell von der engen Verkopplung der Produktionsstrategien der Konzerne mit der Nachfragepolitik des staatlichen Fernmeldemonopols bestimmt. 
Die traditionelle Struktur des BRD-Fernmeldemarktes im Überblick

\begin{tabular}{|c|c|c|c|c|}
\hline Produktgruppe & $\begin{array}{l}\text { Nachfrage- } \\
\text { anteil DBP }\end{array}$ & Anbieter & $\begin{array}{r}\text { Markt- } \\
\text { anteil } \\
\end{array}$ & Besonderheiten \\
\hline $\begin{array}{l}\text { Vermittlungs- } \\
\text { systeme }\end{array}$ & $99,95 \%$ & $\begin{array}{l}\text { Siemens } \\
\text { SEL (ITT) } \\
\text { DeTeWe (Siemens) } \\
\text { T \& N (Bosch) }\end{array}$ & $\begin{array}{l}46 \% \\
30 \% \\
14 \% \\
10 \%\end{array}$ & $\begin{array}{l}\text { Patent- } \\
\text { austausch } \\
\text { zwischen allen } \\
\text { Anbietern }\end{array}$ \\
\hline $\begin{array}{l}\text { Telegrafie- } \\
\text { einrichtungen }\end{array}$ & $100 \%$ & $\begin{array}{l}\text { Siemens } \\
\text { SEL (ITT) }\end{array}$ & $\begin{array}{l}60 \% \\
40 \%\end{array}$ & $\begin{array}{l}\text { Zulieferung von } \\
\text { Siemens an SEL } \\
\text { in erheblichem } \\
\text { Umfang }\end{array}$ \\
\hline $\begin{array}{l}\text { Endgeräte } \\
\text { (einfacher } \\
\text { Hauptanschluß) }\end{array}$ & $100 \%$ & $\begin{array}{l}\text { Siemens } \\
\text { SEL (ITT) } \\
\text { T \& N (Bosch) } \\
\text { DeTeWe (Siemens) } \\
\text { sowie } 8 \text { "Nachbau- } \\
\text { firmen« (Krone, } \\
\text { Bosse, DFG, } \\
\text { Merk, Hagenuk, } \\
\text { Apparatebau Hei- } \\
\text { bel, Telefonfabrik } \\
\text { Reiner, Widmaier) }\end{array}$ & & $\begin{array}{l}\text { Die vier größten } \\
\text { Firmen vergeben } \\
\text { als »Systemführer« } \\
\text { Nachbaurechte } \\
\text { an die kleineren } \\
\text { Firmen }\end{array}$ \\
\hline $\begin{array}{l}\text { Nebenstellen- } \\
\text { anlagen }\end{array}$ & $19 \%$ & $\begin{array}{l}\text { DBP } \\
\text { Private } \\
\text { (wie Anbieter } \\
\text { Endgeräte, aber } \\
\text { keine System- } \\
\text { führerschaften) }\end{array}$ & $\begin{array}{l}19 \% \\
81 \%\end{array}$ & $\begin{array}{l}\text { DBP vermarktet } \\
\text { kleinere Neben- } \\
\text { stellenanlagen } \\
\text { in eigener } \\
\text { Regie }\end{array}$ \\
\hline $\begin{array}{l}\text { Übertragungs- } \\
\text { technik (Weit- } \\
\text { verkehrseinrich- } \\
\text { tungen, Verstär- } \\
\text { kerstellen, Richt- } \\
\text { funkeinr. etc.) }\end{array}$ & $80 \%$ & $\begin{array}{l}\text { Siemens } \\
\text { SEL (ITT) } \\
\text { AEG } \\
\text { TeKaDe } \\
\text { (Philips) }\end{array}$ & & $\begin{array}{l}\text { Systemführer: } \\
\text { Siemens }\end{array}$ \\
\hline Fernmeldekabel & $80 \%$ & $\begin{array}{l}\text { Siernens } \\
\text { AEG } \\
\text { SEL (ITT) } \\
\text { Kabelmetall } \\
\text { sowie } 13 \text { kleinere } \\
\text { Firmen }\end{array}$ & k.A. & $\begin{array}{l}\text { Hersteller } \\
\text { in Rationalisie- } \\
\text { rungskartell } \\
\text { zusammen- } \\
\text { geschlossen. Kein } \\
\text { Systemführer. }\end{array}$ \\
\hline
\end{tabular}

Quelle: Monopolkommission 1981

1 bis 1981 , heute $z . T$. Bosch 
Die traditionelle Struktur der fernmeldetechnischen Beschaffungskartelle im Bereich der Deutschen Bundespost verdeutlicht dies (vgl. die nebenstehende Übersicht). Die DBP hat in den wichtigsten Bereichen des kommunikationstechnischen Binnenmarktes ein mehr oder weniger vollständiges Nachfragemonopol inne und deckt bis in die Gegenwart »nahezu ihren gesamten Bedarf « an fernmeldetechnischen Ausrüstungsgütern auf dem Inlandsmarkt (Ifo 1983, S. 147). Ausländische Anbieter finden in den betreffenden Marktbereichen nur Berücksichtigung, wenn sie selbständige Tochtergesellschaften in der BRD unterhalten. Diese sind fest in die Lieferantenkartelle bei der DBP integriert.

Marktführer in fast allen Bereichen ist der Siemens-Konzern, der zusammen mit AEG und den ausländischen »Bündnispartnern« ITT (SEL) und Philips (PKI) traditionell den Fernmeldemarkt der BRD beherrscht. Die AEG hat durch die Abtrennung der »Telematik «Tochterunternehmen Telefonbau und Normalzeit (T\&N) und ANT-Weitverkehrstechnik während der Konzernkrise Anfang der achziger Jahre entscheidende Marktanteile in den Beschaffungskartellen bei der DBP an den Bosch-Konzern verloren.

In diese stabile Oligopolstruktur sind neben den Großkonzernen eine Reihe von mittelständischen Unternehmen inkorporiert (bei einfachen Fernmeldeendgeräten und im sog. „Kabelkartellk, vgl. Hoppmann/Schlögl 1971 und Raabe/Stöhr 1974), denen die Vermarktung ihrer Produktion durch die Bundespost eine relativ sichere Konkurrenzstellung garantiert und den Erhalt eines aufwendigen Vertriebs- und Marketingapparates entbehrlich macht (vgl. Rittershofer 1977 und ZVEI 1983).

Das entscheidende Element dieses Systems staatlich vermittelter Konkurrenzregulierung bildet die Verzahnung der Auftragsvergabepolitik der Bundespost mit der Entwicklung und Festlegung von marktabgrenzenden Tecbnologienormen. Wie aus der nebenstehenden, nach Angaben der Monopolkommission zusammengestellten, Übersicht hervorgeht, sind die wesentlichen Herstellerkonzerne - allen voran Siemens - als »Systemführer« zugleich direkt an der Ausarbeitung der technischen Entwicklungsnormen für Fernmeldeanlagen beteiligt. Die Erarbeitung dieser Normen erfolgt in "technischen Arbeitskreisen « der Bundespost gemeinsam mit dem Fachverband Fernmeldetechnik des Zentralverbandes der Elektroindustrie (ZVEI) und gelegentlich auch unter Beteiligung einzelner Unternehmen der Fernmeldeindustrie (Monopolkommission 1981, S. 52).

Diese Entwicklungsnormen werden - in verschiedenen Abstufungen (vgl. Dohmen 1980, S. 24) - den betrieblichen Erfordernissen der Bundespost entsprechend auf eine allgemeinverbindliche Standardisierung ausgerichtet (sog. »Fernmeldeeinheitstechnik «) und determinieren als »Marktzugangsnormen in entscheidender Weise die Struktur der Fernmeldemärkte in der Bundesrepublik. Grundsätzlich haben Abweichungen einzelner Hersteller von den Standards der Einheitstechnik zu Folge, daß die Zulassung des betreffenden Geräts durch die Bundespost verweigert wird. »Dies bewirkt ein Ausscheiden des Herstellers aus dem Markt« (Monopolkommission 1981, S. 44).

Die bemerkenswerte Stabilität der Konkurrenz- und Regulierungsmechanismen in der bundesdeutschen Fernmeldeindustrie beruht auf der weitgehenden ökonomischen Dekkung der unternehmenspolitischen Ziele des Staatsbetriebes Bundespost mit den produktions- und marktökonomischen Strategien der Fernmeldekonzerne.

Ökonomisch »erfolgreich « ist dieser staatlich -industrielle Regulierungsverbund in doppelter Hinsicht. Einerseits waren die in diesem Rahmen getroffenen frühzeitigen und langfristigen Entscheidungen über den Einsatz fortgeschrittenster Fernmeldetechnologien eine entscheidende Voraussetzung dafür, daß in der BRD im Verlauf der Nachkriegszeit ein 
Fernmeldenetz entstehen konnte, dessen Entwicklungsniveau heute als international vorbildlich gilt. Kein anderes Industrieland verfügt heute über eine Fernmeldeinfrastruktur von vergleichbarer technischer Geschlossenheit und Einheitlichkeit (vgl. Möhring 1976, S. 39 f., sowie Wirtschaftswoche v. 8.2.85).

Andererseits ist im hohen Perfektionsgrad der westdeutschen Fernmeldeeinheitstechnik der entscheidende Konkurrenzvorteil der westdeutschen Fernmeldeindustrie beim Export nachrichtentechnischer Ausrüstungsgüter auf dem Weltmarkt zu sehen. Die Exportstrategie der BRD-Konzerne basiert im wesentlichen auf der Lieferung »schlüsselfertiger« fernmeldetechnischer Großsysteme, oftmals kompletter nationaler Fernmeldenetze. Primäres Entwicklungsziel ist nach eigener Darstellung »nicht Modernität um jeden Preis, sondern ein Angebot ausgereifter technologischer Lösungen mit optimalem Nutzen für Anwender und Betreiber privater und öffentlicher Kommunikationsnetze (ZVEI 1983, S. 57). Die staatlich-industrielle Technologienormenpolitik im Rahmen der fernmeldetechnischen Beschaffungskartelle bei der DBP hat somit wesentliche Voraussetzungen dafür geschaffen, daß die Fernmeldeindustrie zu einem der wichtigsten Exportzweige des elektrotechnischen Anlagenbaus in der BRD werden konnte (vgl. Plettner 1983).

\section{Neuformierung der Konkurrenzverhältnisse in der westdeutschen Telekommunikationsindustrie}

Wie wirken sich nun der Bruch der traditionellen Branchenstruktur und die Internationalisierungstendenzen in der Produktion auf die Konkurrenzstrategien des Branchenkapitals in der westdeutschen Telekommunikationsindustrie aus? Welche Rolle kommt den traditionellen Mechanismen der Konkurrenzregulierung in der Fernmeldeindustrie in den Krisenstrategien des bundesdeutschen Elektronikkapitals zur Bewältigung dieses Strukturbruches zu?

Das strategische Problem der westdeutschen Konzerne im internationalen Konkurrenzwettlauf um die Zukunftsmärkte der IuK-Technik liegt, wie bereits dargestellt, in der relativen Schwächeposition im engeren Bereich der EDV-Produktion. »Ein zu kleiner Heimatmarkt, der zudem maßgeblich von Tochtergesellschaften ausländischer Firmen beherrscht wird, ein fragmentierter europäischer Markt, ... eine zu geringe Präsenz auf dem Weltmarkt« sowie »eine ungenügende Arbeitsteilung in der deutschen informationstechnischen Wirtschaft « werden von der Industrie als wesentliche Ursachen dieser Situation angeführt (Memorandum 1983, S. 8).

Die relative Konkurrenzschwäche im EDV-Bereich kontrastiert mit der Exportstärke der Elektronikindustrie in Produktionszweigen, in denen die Verknüpfung der Informationstechnik mit Mechanik, Nachrichtentechnik und anderen Produktionstechnologien für "anwendungsbezogene Systemlösungen im Vordergrund steht (ebd., vgl. auch BMFT 1984, S. 22). Die informationstechnische Industrie hebt die waus den Besonderheiten des Heimatmarktes sich ergebenden "Konkurrenzvorteile hervor: "Langfristige Systemplanung in Zusammenarbeit mit den Anwendern ermöglicht die evolutionäre Weiterentwicklung informationstechnischer Problemlösungen«. Auf diesem Hintergrund wird »der Heimatmarkt höchste Bedeutung für Innovation und deren Erprobung behalten. In diesem Rahmen wird der Ausbau der informationstechnischen Netze und Dienste durch die Bundespost eine entscheidende Rolle spielen. Hinreichende Seriengrößen in der Komponen- 
ten- und Geräteproduktion sind bei schärfer werdendem Preiswettbewerb unabdingbar « (Memorandum 1983, S. 9).

Solche Formulierungen in einem industriepolitischen »Grundsatzprogramm« der im Bereich der IuK-Technik maßgebenden bundesdeutschen Unternehmen reflektieren das strategische Interesse dieser Kapitalgruppierung an seiner Ausdehnung der traditionellen »Leitfunktion" des fernmeldetechnischen Binnenmarktes auf die im Zuge der Integration von EDV- und Nachrichtentechnik entstehenden neuen Telekommunikationsmärkte. Die zur Realisierung der produktionsökonomisch erforderlichen Massenproduktionseffekte bei der Herstellung elektronischer Telekommunikationssysteme müssen durch eine solche staatlich forcierte und regulierte Binnenmarktexpansion sichergestellt werden. Ebenso kommt der Bundespost im Hinblick auf die verschärfte Technologienormenkonkurrenz eine Vorreiterfunktion im internationalen Standardisierungswettlauf zu (vgl. ebd., S. 11).

Es verwundert nicht, wenn in diesem Zusammenhang die Forderung erhoben wird, daß $» z u$ mindest in Kermbereichen der IuK-Technik Beschaffungsvorhaben grundsätzlich in Zusammenarbeit mit inländischen Herstellern entwickelt und abgewickelt werden" sollen (ebd., S. 13). Hiermit ist von seiten des bundesdeutschen Elektronikkapitals zugleich die Demarkationslinie gegenüber etwaigen Deregulierungsbestrebungen im bundesdeutschen Telekommunikationswesen gezogen.

Freilich bleibt ein Modernisierungsbündnis des nationalen Branchenkapitals in einer Situation zugespitzter ökonomischer Krisenentwicklung nicht unberührt von kapitalinternen Interessenwidersprüchen. Konflikte und Brüche entstehen, wenn ökonomische Differenzen innerhalb eines solchen kapitalistischen Interessenblocks und gegenüber anderen Kapitalgruppierungen zum Ausgleich gebracht werden müssen, wenn Strukturkrisen ganzer Konzerne das Auseinanderbrechen der Interessenkoalition heraufbeschwören oder wenn die Abschottung des nationalen Binnenmarktes Protektionismusvorwürfe von Seiten internationaler Konkurrenz hervorruft. Einige wesentliche Konflikte seien hier skizziert. Festzuhalten ist zunächst, daß das naheliegende Interesse der nationalen Elektronikindustrie in der BRD an der Aufrechterhaltung der Regulierungsfunktion des staatlichen Fernmeldemonopols kaum von potentiellen Anwendern und Betreibern neuer Kommunikationstechniken in Frage gestellt wird (z.B. von Banken, Versicherungs- oder Industrieunternehmen anderer Branchen). Hierin unterscheiden sich die kapitalinternen Interessenskonstellationen in der BRD zumindest der Form nach von denen etwa in den USA oder in Großbritannien, wo einflußmächtige Organisationen der »Telekommunikationsbenutzer zu den wichtigsten Exponenten der »Deregulierungslobby« gehörten. Ein wesentlicher Grund dafür, daß solche Interessengegensätze in der BRD bislang kaum zu einer ernsthaften Infragestellung der Regulierunsfunktion des staatlichen Fernmeldemonopols geführt haben, ist sicherlich in dem Umstand zu suchen, daß die wichtigsten Nutzer neuer Telekommunikationsnetze und -dienste, allen voran die bundesdeutschen Großkonzerne des Bank- und Versicherungskapitals, eng mit den Konzernen der Herstellerindustrie verbunden sind. Die Telekommunikationshersteller Siemens, Bosch, Nixdorf sowie seit der jüngst erfolgten Übernahme durch Daimler-Benz auch die AEG sind durch vielfältige Kapital- und Personalbeziehungen mit den führenden Gruppen des bundesdeutschen Finanzkapitals, der Deutschen Bank und der Allianz AG funktional verflochten (vgl. Lüthje 1985, S. 89 ff., sowie Spiegel v. 11.2.85). Akut gefährdet war das Modernisierungsbündnis der westdeutschen Kommunikationsunternehmen auf dem Höhepunkt der AEG-Krise im Jahre 1982. Die AEG sollte nach Sanierung der defizitären Produktionszweige im Konsumgüterbereich in einen »Telematik«-Konzern um- 
gewandelt werden (vgl. z.B. FR v. 15.9.1985). Durch die Übernahme der AEG-Tochtergesellschaften T \& N und ANT durch Bosch sowie Mannesmann und die Allianz AG wurde dieses sog. »Telematik-Konzept « faktisch unter Führung des Bosch-Konzerns verwirklicht, der so zum zweitwichtigsten Hersteller yon Telekommunikationseinrichtungen in der BRD avancierte (vgl. Wirtschaftswoche v. 4.12.84).

Auf diese Weise gelang zugleich ein entscheidender Schritt zur langfristigen Absicherung des Telekommunikationsmarktes in der BRD. Das ursprüngliche » Telematik-Konzept« zur Sanierung der AEG sollte bekanntlich durch eine Kapitalbeteiligung des britischen Elektround Fernmeldekonzerns GEC ermöglicht werden. Einer der bedeutenden Konkurrenten der bundesdeutschen Konzerne auf dem Weltmarkt hätte so Zugang zu den Beschaffungsund Technologiekartellen im Bereich der Bundespost und damit zur Technologieentwicklung aller westdeutschen Telekommunikationshersteller erhalten. An der Vermeidung eines solchen Präzedenzfalles durch eine "nationale Lösung« der AEG-Krise hat deshalb nicht zuletzt Siemens ein vitales Interesse gehabt (vgl. NZZ v. 10.1.82 und Spiegel v. 5.7.82).

Konnte es den Unternehmen der bundesdeutschen Telekommunikationsbranche bislang gelingen, ihr Modernisierungsbündnis gegenüber Interessenwidersprüchen zu anderen Kapitalfraktionen und dem Vordringen ausländischer Konkurrenten in den Kernbereich des Telekommunikationsbinnenmarktes abzuschotten, so ist gleichwohl absehbar, daß die traditionelle Verteilung der "Einflußsphären« zwischen den Konzernen im Rahmen der postalischen Beschaffungs- und Technologiekartelle längerfristig nicht aufrechtzuerhalten ist. Einerseits werden im Zuge des Zusammenwachsens von EDV- und nachrichtentechnischer Produktion bestehende Marktabgrenzungen u.a. im Endgerätebereich (z.B. zwischen einfachen Telefongeräten, Nebenstelleneinrichtungen, Teletex-, Telefaxterminals etc.) obsolet, andererseits stehen die Kartellstrukturen in der bundesdeutschen Fernmeldeindustrie angesichts der beschleunigten Internationalisierung der Telekommunikationsmärkte unter verschärftem Druck ausländischer Konkurrenten. Ein charakteristisches Beispiel für die aus dem Zusammenwachsen der kommunikationstechnischen Produktionsmärkte sich ergebenden Interessenüberschneidungen zwischen "alten « und "neuen « Telekommunikationsherstellern bietet die Entwicklung des Nixdorf-Konzerns. Bis vor wenigen Jahren noch ein "Outsider « in der Telekommunikationsbranche ist dieser inzwischen in den Rang eines etablierten Lieferanten der Bundespost aufgerückt. Nach längeren Konflikten gelang Nixdorf 1984 mit der Zulassung eines Digitaltelefons durch die Bundespost der Vorstoß in den von etablierten Lieferfirmen wie Siemens und T \& $N$ dominierten Endgeräte- und Nebenstellenmarkt. Nixdorf hat 1984 angekündigt, im Rahmen seiner längerfristigen Konzernplanung umfangreiche Investitions- und Entwicklungsprogramme im Bereich der kommunikationstechnischen Produktion vorzunehmen. Die etwa zum gleichen Zeitpunkt erfolgte Börseneinführung der Nixdorf-Aktie durch die Deutsche Bank dient u.a. zur Abdeckung des dafür erforderlichen Finanzbedarfs (vgl. FAZ v. 21.2.8)

Ein weiteres Beispiel für die Schwierigkeiten der etablierten Telekommunikationshersteller in der BRD, die traditionelle Regulierung des Binnenmarktes aufrechtzuerhalten, bildet das Ende des seit den sechziger Jahren bestehenden Rationalisierungskartells in der Kabelindustrie (vgl. Hoppmann/Schlögl 1971 und Raabe/Stöhr 1974). Die mittelständischen Hersteller dieser Branche sind nicht mehr in der Lage, die Investitionen für die zukünftige Glasfaserkabeltechnologie zu tätigen. Das Bundeskartellamt hat inzwischen das Auslaufen der Genehmigung für das »Kabelkartell« beschlossen (vgl。SZ v。 4.1.85).

Durch die Errichtung einer gemeinsamen Produktionsstätte für Glasfaserkabel mit Absatz- 
garantien der Bundespost versuchten die fünf Großunternehmen der bundesdeutschen Kabelbranche (Siemens, AEG, SEL, PKI und Kabelmetall), die traditionelle Abschottung des Binnenmarktes weiterhin zu gewährleisten. Dieses Projekt mußte allerdings vom Bundeskartellamt untersagt werden, weil Ende 1983 das Chemieunternehmen Wacker gemeinsam mit dem kurz vor der Entflechtung stehenden US-Konzern AT \& T ebenfalls den Aufbau einer Glasfaserkabelproduktion in der BRD ankündigte (vgl. Wirtschaftswoche v. 13.1.8 und Spiegel v. 4. 12. 85). Nach der Ablehnung des Bundeskartellamtes hat Siemens gemeinsam mit der US-Gesellschaft Corning den Aufbau einer Glasfaserkabelproduktion in der BRD nunmehr in eigener Regie in Angriff genommen - mit der ausdrücklichen Option allerdings, daß zu einem späteren Zeitpunkt noch weitere Gesellschafter aufgenommen werden können (vgl. FR v. 4. 12.84). Der Fall des »Kabelkartells« illustriert augenfällig, wie die internen Mechanismen der Konkurrenzregulierung in der Telekommunikationsindustrie der BRD unter den Zwängen des zugespitzten Konkurrenzkampfes auf dem Weltmarkt brüchig werden können.

Nicht mehr auszuschließen ist inzwischen auch eine offene handelspolitische Konfrontation zwischen der BRD und den USA, vergleichbar mit der Anti-Protektionismuskampagne der USA gegen die restriktiven Importbedingungen für Telekommunikationseinrichtungen in Japan (vgl. z.B. FT v. 29.3. 85). Der IBM-Konzern tritt in letzter Zeit mit deutlicher Kritik an den Beschaffungspraktiken der europäischen Fernmeldeverwaltungen in die Öffentlichkeit und fordert eine Beschränkung der marktregulierenden Funktionen der staatlichen Fernmeldemonopole auf den Netzbereich und eine Liberalisierung der Endgerätemärkte(vgl. FAZ v. 24.10.85 und 28.9.85).

Das »Protektionsdilemmak der bundesdeutschen Telekommunikationsindustrie ist nicht zu übersehen. Der Amortisationszwang für die gesteigerten Kapitalaufwendungen in der kommunikationstechnischen Produktion macht die Ausdehnung der Anteile auf den Kommunikationsmärkten der Industrieländer und hier u.a. auf dem liberalisierten US-Markt auch für die betreffenden bundesdeutschen Unternehmen zu einer Existenzfrage. Eine Liberalisierung des Telekommunikationsmarktes in der BRD durch eine partielle Deregulierung des Fernmeldemonopols der Bundespost ist die Gegenleistung, die im Interesse der Aufrechterhaltung der Exportchancen der nationalen Telekommunikationsindustrie von bundesdeutscher Seite auf mittlere Sicht zu erbringen ist. Eine Konsequenz, die den unmittelbaren Konkurrenzinteressen einiger betroffener Einzelkapitale zuwiderlaufen mag, aber der Sachzwanglogik einer weltmarktorientierten Modernisierungspolitik entspricht.

\section{Umstrukturierung der Bundespost als Krisenlösungsprogramm}

Bis hierher haben wir zentrale ökonomische Aspekte der Regulierungskrise im Telekommunikationssektor der BRD dargestellt. Abschließend soll nun gefragt werden, in welcher Form sich der Strukturbruch in den Konkurrenzbedingungen des Kapitals in der Infrastrukturund Regulierungspolitik des Staates reproduziert.

Zugespitzt läßt sich die These formulieren, daß die relative Stabilität des staatlichen Fernmeldemonopols in der BRD durch die bislang erfolgreiche kapitalinterne Regulierung der Strukturkrisen in der Telekommunikationsbranche ermöglicht wurde. Die ungebrochene Konkurrenzstärke der elektrotechnischen Universalkonzerne der BRD auf dem Fernmeldeweltmarkt bildet im Zusammenhang einer Interessenallianz der dominanten Gruppierun- 
gen des westdeutschen Exportkapitals die Voraussetzung dafür, daß sich die ökonomischen Strukturprobleme im »Zukunftssektor Telekommunikation (insbesondere die Wettbewerbsschwäche bundesdeutscher Konzerne in der Computer- und Halbleiterproduktion) bislang nicht zu einer offenen Formkrise staatlicher Interventions- und Regulierungspolitik verdichtet haben.

Die Strategie staatlicher Industriepolitik im Telekommunikationsbereich hat sich als außerordentlich stabil erwiesen und ist auch im Gefolge der konservativ-liberalen Regierungsübernahme von $1982 \mathrm{kaum}$ modifiziert worden. Über die Aufrechterhaltung eines staatlichen Monopols im Kermbereich des Kommunikationsnetzes besteht prinzipiell politischer Konsens zwischen Unternehmern (vgl. z.B. DIHT 1983, S. 79 ff.; ZVEI 1983 u.a.m.), politischen Parteien (vgl. z.B. SPD-Medienkommission 1984, CDU 1981, FDP 1984) und Gewerkschaften (vgl. z.B. Bock 1985). Wenn die Bundespost als »Motor « nationaler Industriepolitik im Telekommunikationssektor fungieren soll, so ist damit nicht nur der Ausbau der traditionellen »Investitionslenkungsfunktion « des Posthaushaltes (der immerhin ein Fünftel aller industriellen Anlageinvestitionen in der BRD trägt; vgl. Rittershofer 1977 sowie DP vom 20.10.85), sondern auch eine bestimmte politische Strategie zur Durchsetzung dieses großtechnologischen Investitionsprogrammes intendiert. Die $\gg$ Modernisierung der Volkswirtschaft « im Telekommunikationsbereich soll durch den stillschweigenden Konsens der dominanten Fraktionen des westdeutschen Exportkapitals und jenes schrumpfenden Kerns der Lohnabhängigen, denen die beschleunigte Durchsetzung neuer IuK-Techniken relative Arbeitsplatzsicherheit verheißt, abgestützt werden (vgl. Hauff 1979).

De facto besteht in der BRD eine nationale Modernisierungskoalition von Kapital, Gewerkschaft und Staat im Kommunikationswesen, denn auch von den Gewerkschaften wird die Orientierung staatlicher Infrastrukturpolitik am Sachzwang der internationalen Konkurrenzfähigkeit der bundesdeutschen Industrie prinzipiell in Frage gestellt (vgl. Rittershofer 1977, Bock 1985). Insofern ließe sich schlußfolgern, daß das staatliche Fernmeldemonopol in der $\mathrm{BRD}$ auch deshalb gegen neo-konservative Privatisierungsforderungen weitgehend immun bleiben konnte, weil staatliche Regulierung der Infrastrukturpolitik im Bereich der neuen IuK-Techniken deren »sozialfriedliche« Durchsetzung erleichtert.

Wie sieht das zukünftige Szenario der Interventionspraxis des Staates im Rahmen einer solchen, an den Konkurrenzinteressen des »Exportkerns« des westdeutschen Kapitals ausgerichteten Industriepolitik im Telekommunikationsbereich aus?

(a) Zentrale Aufgabe des Staates ist und bleibt der Ausbau der kommunikationstechnischen Netzinfrastruktur. Festgehalten wird an der traditionellen Gleichschaltung der Investitionspolitik der Bundespost und der Fernmeldekonzerne. Die mittelfristige Investitionsplanung der DBP ist die Grundlage der Investitions- und Personalplanung der beteiligten Unternehmen (Ifo 1983, S. 84). Die Ausbauplanung des ISDN-Netzes ${ }^{2}$ soll dabei nach Auffassung industrienaher Experten ausdrücklich nicht dem bei Fernmeldeinvestitionen der Bundespost traditionell gepflegten Prinzip der »Bedarfsorientierung" folgen (vgl. Arnold 1984, S. 12). Die Infrastrukturinvestitionen der DBP werden auf die Förderung exportrelevanter Schlüsseltechnologien konzentriert, d.h. der Ausbau des ISDN-Netzes für die Daten-, Text- und Sprachkommunikation besitzt unbedingte Priorität vor dem Ausbau z.B. des Kabelfernsehnetzes (d.h. der sog. »Kupferverkabelung «, vgl. Ifo 1983, S. 88). Die vieldiskutierte Fernseh verkabelung soll nach Kriterien des effektiven wirtschaftlichen Bedarfs erfolgen, wobei eine finanzielle Entlastung der DBP dadurch erreicht werden kann, »daß die Errichtung von Inselnetzen von privaten Interessenten durchgeführt wird« (ebd. S. 89). Die beträchtlichen finan- 
ziellen Überschüsse der Bundespost der letzten Jahre bilden die ökonomische Grundlage dieses "Zukunftsinvestitionsprogramms«, das bis zum abgeschlossenen Ausbau eines Glasfaseruniversalnetzes (etwa in den Jahren 2010 bis 2020) die astronomische Größenordung von 300 Mrd. DM erreichen dürfte (so eine Schätzung der Enquête-Kommission Neue IuK-Techniken des Bundestages 1983, S. 229).

(b) Im Widerspruch zu einer solchen »Kurzschaltung«staatlicher und industrieller Investitionspolitik beim forcierten Ausbau des Telekommunikationsnetzes steht jedoch die Internationalisierung der Konkurrenz in der Telekommunikationsindustrie. Hierdurch besteht mittlerweile auch in der BRD akuter Druck zur Deregulierung des nationalen Kommunikationsmarktes. Entsprechende politische Entscheidungen bereitet derzeit eine von der Bundesregierung eingesetzte»Regierungskommission Fernmeldewesen « vor (mit Vertretern von Parteien und Unternehmerverbänden, Wissenschaftlern sowie einem Repräsentanten der Postgewerkschaft).

Das sich gegenwärtig abzcirhnende Regulierungsmodell für den bundesdeutschen Telekommunikationsmarkt läßt sich in etwa wie folgt umreißen:

- Der Kernbestand des Fernmeldemonopols, die Hoheit der DBP über die Kommunikationsnetze, bleibt weitgehend erhalten. In diesem Punkt besteht seit je bemerkenswerte Übereinstimmung zwischen neo-liberalen Kritikern des Fernmeldemonopols (vgl. z.B. Mestmäcker 1980, v. Weizsäcker 1983 sowie mit Einschränkungen Monopolkommission 1981) und den Befürwortern einer technokratisch-pragmatischen Industriepolitik (vgl. z.B. Ifo 1983, Arnold 1984). Nicht auszuschließen ist jedoch die Zulassung privater Anbieter beim Betrieb von sog. »Mehrwertnetzen« (value added networks, VAN), wie etwa im jüngsten Jahresgutachten des Sachverständigenrates gefordert wurde (vgl. FAZ v. 26.11.85).

- Die Beschaffungspolitik der DBP wird partiell für ausländische Unternehmen und für Telekommunikationshersteller aus dem Bereich der EDV-Industrie geöffnet. Dies ist auf neuen Telekommunikationsmärkten, z.B. bei Datenübermittlungseinrichtungen, Bildschirmtext (Auftragsvergabe an IBM) oder den Breitbandpilotversuchen der Bundespost (Beteiligung z.B. von Nixdorf) schon seit einiger Zeit der Fall (vgl. Tenzer 1985).

- Längerfristig wird das staatliche Fernmeldemonopol auf den Bereich der Kommunikationsnetze beschränkt, der Post wird ihr Anbietermonopol bzw. ihre regulierende Marktstellung (als Zulassungsbehörde) im Bereich der kommunikationstechnischen Endgeräte u.U. auch beim einfachen Fernsprechanschluß - entzogen.

Mit dieser "neuen Arbeitsteilung zwischen Post und Privatanbietern (vgl. FAZ v. 28.9.1985) wäre auch den strategischen Interessen der etablierten Telekommunikationshersteller in der BRD Rechnung getragen, indem die Ausrüstung des Telekommunikationsnetzes mit Schlüsselsystemen der Übertragungs-, Vermittlungs- und Kabeltechnologie regulierter Bereich bliebe und die Bundespost hier auch weiterhin entscheidend an der Festlegung exportrelevanter Technologiestandards beteiligt wäre. Die Deregulierung des besonders wachstumsträchtigen Endgerätemark‡es würde auch ausländische Konkurrenten den Zugang zum bundesdeutschen Kommunikationsmarkt ermöglichen und umgekehrt zur langfristigen Absicherung der Exportchancen westdeutschen Hersteller auf den Märkten anderer Industrieländer beitragen.

(c) Die "sektorspezifische« Konzentration der Investitionsmittel der Bundespost auf den strategischen Kernbereich der neuen IuK-Netze wird ökonomisch abgesichert durch die umfassende Ausrichtung der Unternehmenspolitik der DBP am betriebswirtschaftlichen Kalkül der Gewinnorientierung anstelle der traditionellen Verpflichtungen zur »Kostendeckung« 
(Kalkulation auf Basis von Marktpreisen statt allgemeinverbindlicher Gebühren). Nicht also Privatisierung ökonomischer Staatsfunktionen im orthodox neo-liberalen Sinne, sondern deren durchgreifende „Kommerzialisierung « bei weitgehender Aufrechterhaltung staatlicher Hoheitsrechte ist angesagt (zum Begriff »commercialism« vgl. z.B. die Darstellung der Entwicklung der British Post Office bei Batstone u.a. 1984, S. 26 ff.).

Die soziale und politische "Achillesferse des großindustriell-staatlichen Modernisierungsprogramms im Telekommunikationssektor der BRD sind die sog. »Quersubventionen« innerhalb des Gesamtunternehmens Bundespost, d.h. korrekt ausgedrückt der Umstand, daß die DBP betriebswirtschaftliche Kostendeckung erzielt, indem die Verluste in den Postdiensten (Brief-, Paketverkehr etc.) durch die Gewinne aus dem Fernmeldebereich bilanziell ausgeglichen werden. Um die »Überforderung des Fermeldewesens durch das Postwesen " (so eine blumige Formulierung des DIHT 1983, S. 79) zu beseitigen, steht zur Diskussion, die Bundespost in drei voneinander unabhängige Unternehmensbereiche aufzugliedern (Fernmelde-, Post- und Postbankdienste), die jeweils zur Gewinnerzielung aus eigener Kraft verpflichtet sind (vgl. z.B. Wirtschaftswoche v. 8.9.84 sowie 15.2.85, SVR 1985).

Wenn auch die konkrete Gestalt solcher Pläne gegenwärtig noch unbestimmt ist, so erfordert der Abbau der "Quersubventionen « weitreichende Rationalisierungs- und Umstrukturierungsmaßnahmen im Bereich der Postdienste, die nach heute vorliegenden Gutachten privater Unternehmensberatungsfirmen die Vernichtung von jährlich 20000 bis $30000 \mathrm{Ar}$ beitsplätzen innerhalb von sechs bis acht Jahren zur Folge haben könnten (vgl. Handelsblatt v. 11.5.85 sowie Knight Wendling AG 1985).

Die »Entflechtung (Wirtschaftswoche v. 15.2.85) der traditionellen Struktur des Staatsunternehmens Bundespost ist darüber hinaus eine notwendige Rahmenbedingung für die weitere $\mathrm{Be}$ teiligung der Bundespost im deregulierten Bereich des Fernmeldemarktes (Endgeräte und Nebenstellen) »unter Wettbewerbsbedingungen«, wird doch die Verbindung von kommerziell agierenden Unternehmensteilen und Aufgabenbereichen staatlicher "Daseinsvorsorge" mit Monopolcharakter in einem Unternehmen nach herrschender wettbewerbsrechtlicher Meinung als unvertretbar angesehen (vgl. z. B. Mestmäcker 1980, Monopolkommission 1981).

\section{Modernisierung der Telekommunikation und Gewerkschaften}

Vor dem Hintergrund der heute auch in der BRD wirksamen Tendenzen zu einer Liberalisierung des Fernmeldemonopols scheint sich unsere eingangs aufgestellte These zu bestätigen, daß der "Entstaatlichung « des Telekommunikationswesens in den kapitalistischen Industrieländern ein allgemeiner ökonomischer Strukturzwang aufgrund der krisenhaften Veränderungen in den Produktions- und Akkumulationsbedingungen des Kapitals unterliegt. Die vom Diktat der veränderten Konkurrenzverhältnisse auf dem Weltmarkt angetriebene Neubestimmung der Grenzen der Staatsintervention im Telekommunikationssektor soll in der BRD nicht nach dem Muster einer harten neo-liberalen »deregulation policy«, d.h. im Wege einer radikalen Privatisierung ökonomischer Staatsfunktionen, vollzogen werden (wie in Großbritannien); vielmehr zeichnet sich eine grundlegende Neudefinition der ökonomiscben Rationalitätskriterien kapitalistischer Staatsintervention ab, die sich in der Abkehr vom ökonomischen Prinzip der »Daseinsvorsorge« und der Durchsetzung einer konsequenten Gewinn- und Kostenorientierung des Staatsunternehmens Bundespost manifestiert. Dieser Umstrukturierungsprozeß ließe sich (in Anlehnung an Batstone u.a. 198) als Kommerzia- 
lisierung ökonomischer Staatsfunktionen charakterisieren: Die Bundespost, so könnte man es zugespitzt formulieren, soll zwar ein Staatsunternehmen bleiben, sich aber wie ein Privatunternehmen verhalten.

Welches sind die sozialen und politischen Gefahren einer solchen an den Stukturzwängen kapitalistischer Weltmarktkonkurrenz ausgerichteten Modernisierungsstrategie, und welche Probleme stellen sich den Gewerkschaften?

Hier ist nicht nur auf die enormen gesarntwirtschaftlichen Rationalisierungsfolgen und den Arbeitsplatzabbau bei der Bundespost durch den Ausbau neuer Telekommunikationsnetze hinzuweisen (stellvertretend für viele Zerdick 1984, Kubicek/Rolf 1985). Die Umstrukturierung des Staatsunternehmens Bundespost würde auch das politische Terrain des gesellschaftlichen Konkflikts um die Anwendung und Kontrolle der neuen IuK-Technologien entscheidend verändern. Die Kommerzialisierung der DBP dient im Kalkül kapitalistischer Krisenbewältigungsstrategie nicht nur der beschleunigten Durchsetzung des ISDN-Investitionsprogramms in ökonomischer Hinsicht, sondern auch dessen politischer Absicherung: "nach außen« gegenüber zunehmenden »Akzeptanzängsten« in der Bevölkerung und unter den rationalisierungsbetroffenen Lohnabhängigen; »nach innen « gegenïber gewerkschaftlichem Widerstand gegen den mit der Modernisierung der Telekommunikation verbundenen Arbeitsplatzabbau bei der DBP. Beide Aspekte dieser politischen Absicherungsstrategie stellen die Gewerkschaften vor Probleme, die das vorherrschende sozialpartnerschaftliche Selbstverständnis der Gewerkschaftspolitik in der BRD in seinen Grundlagen berühren. Einschlägige Stellungnahmen der Spitzenverbände der Wirtschaft verweisen sehr deutlich auf die intendierte "Außenabsicherung" des IuK-technischen Zukunftsinvestitionsprogramms durch den Ausbau des »Präventions- und Sicherheitsstaates« (vgl. Hirsch 1980) im Telekommunikationswesen. "Die Weiterentwicklung des Fernmeldewesens«, so etwa ein führender Vertreter des DIHT, »darf nicht in Abhängigkeit von tages- und parteipolitischen Auseinandersetzungen geraten. Sie darf ebenso nicht von der jeweiligen Lage der öffentlichen Haushalte abhängig werden« (Mayer, 1983). Deshalb, so die Schlußfolgerung, »müssen auch die Voraussetzungen geschaffen werden, daß eine solche Weiterentwicklung unabhängig von den jeweils aktuellen politischen Trends und Konstellationen gesichert ist « (ders. 1986).

Die institutionellen Voraussetzungen für eine solche politische Abschottungsstrategie bestehen, denn die unternehmensrechtliche Eigenständigkeit der DBP garantiert, daß die Entscheidungen über den Posthaushalt (mit z.Zt. etwa $70 \mathrm{Mrd}$. DM größer als der größte Einzeletat des Bundes) faktisch keiner parlamentarischen Kontrolle unterliegen und damit gegenüber instabilen politischen Kräftekonstellationen und »unsicheren « parlamentarischen Mehrheitsverhältnissen abgeschirmt werden können (vgl. Raabe/Stöhr 1974). Darüber hinausgehend streben die Unternehmerverbände heute eine gesetzliche Festschreibung der Kommerzialisierung der Bundespost mittels einer Reform von Postverwaltungs- und Fernmeldeanlagengesetz an. Die Forderungen laufen praktisch auf die strikte Orientierung der Infrastruktur der DBP auf Kriterien der Gewinnorientierung und des ökonomischen Bedarfs der Wirtschaft für Telekommunikationsdienstleistungen hinaus (vgl. Mayer 1986 und BDI, BdA, DIHT u.a. 1986).

Angesichts der ökonomischen Dimensionen und der weitreichenden sozialen Folgewirkungen des Ausbaus der ISDN-Infrastruktur erscheint die Entpolitisierung der hiermit verbundenen gesellschaftlichen Konflikte als eine wesentliche soziale Stabilitätsbedingung des großindustriellen Modernisierungsprogramms in der Telekommunikation. Von Unterneh- 
merseite sind hier unverkennbar Lehren aus den Auseinandersetzungen um andere $\gg$ Großtechnologieprojekte" (wie z.B. um Atomkraftwerke) und aus politischen Konflikten um den Datenschutz (Volkszählung!) gezogen worden; mit Erfolg offenbar, denn eine breite Auseinandersetzung um neue IuK-Technologien gibt es heute in der politischen Öffentlichkeit der BRD faktisch nicht.

Fatal ist eine solche Entpolitisierung v.a. für die von den Rationalisierungswirkungen des Einsatzes neuer Telekommunikationstechniken betroffenen Lohnabhängigen. »Da es derzeit keine erkennbar wirksamen Formen der Mitbestimmung zur Beeinflussung dieser neuen Formen der Rationalisierung durch atomisierte Auslagerung von Arbeit gibt «, stellt z.B. Kubicek (1986) mit Bezug auf das Problem der sog. Heimarbeit zutreffend fest, "wird mit dem Angebot der 'billigen Datenfernübertragung für jedermann' direkt in die betrieblichen Rationalisierungsprozesse zugunsten der Arbeitgeber eingegriffen.« Dasselbe gilt auch für Rationalisierungsmaßnahmen im Bürobereich und in der Produktion (z.B. bei betriebsübergreifenden Verfahren der computergestützten Fertigungs- und Logistikrationalisierung oder bei der Einführung elektronischer Fernsatzverfahren in der Druckindustrie).

Nimmt man die heute schon bestehende politische und institutionelle Abschottung der staatlich-industriellen Technologiepolitik im bundesdeutschen Telekommunikationswesen zur Kenntnis, erscheinen die in letzter Zeit von gewerkschaftlicher Seite verstärkt erhobenen Forderungen nach einer Ausweitung überbetrieblicher Mitbestimmungsrechte beim Einsatz neuer IuK-Technologien (vgl. Bleicher 1985) wenig überzeugend. Im Gegenteil: die auf eine Ausschaltung öffentlicher Kontrolle über das Kommunikationswesen angelegte unternehmerische Modernisierungspolitik ist dazu angetan, eine solche sozialpartnerschaftliche Mitgestaltung durch Mitbestimmung bereits im Vorfeld zu unterlaufen.

Es stellt sich die Frage, welche Ansätze die Gewerkschaften haben, die Rationalisierungsauswirkungen des Ausbaus der neuen IuK-Netze »von innen heraus«, also durch eine entsprechende Beeinflussung der Unternehmenspolitik der Bundespost, zu verhindern oder zu begrenzen.

Die Deutsche Postgewerkschaft spielt in diesem Zusammienhang - nach einem Wort ihres Vorsitzenden K. v. Haaren - in der Tat die Rolle einer »Schlüsselgewerkschaft« innerhalb des DGB. Festzuhalten ist jedoch, daß auch die DPG weder im Rahmen der Mitbestimmungsrechte der Personalräte noch durch ihre Beteiligung am Postverwaltungsrat über nennenswerte formelle Möglichkeiten zur Beeinflussung der postalischen Technologiepolitik verfügt. Die jahrelang erhobene Forderung der DPG nach paritätischer Mitbestimmung im Postverwaltungsrat ist auch in Zeiten sozialdemokratischer Regierungsverantwortung niemals verwirklicht worden.

Bei der Bundespost sind heute die Zeichen deutlich auf die Zurückdrängung des gewerkschaftlichen Einflusses in der Personal- und Beschäftigungspolitik des Unternehmens gestellt. Auf den anstehenden massiven Arbeitsplatzabbau im Postwesen wurde bereits hingewiesen. Nach Aussagen des DPG-Hauptvorstandes ist hier mit der Gefährdung von 30000 Arbeitsplätzen in den nächsten Jahren zu rechnen (vgl. FAZ v. 16.11.85). Eine erste Auswertung einer vom Postministerium in Auftrag gegebene Studie »strategisches Konzept für das Postwesen « (mit der Vorgabe der Eigenwirtschaftlichkeit des Postwesens, vgl. KnightWendling AG 1985) ergab die akute Gefährdung von ca. 55000 Arbeitsplätzen im Post- und Postgirodienst (vgl. DPG Hessen 1985). Die Beratungsfirma sprach sogar von einem Rationalisierungspotential von jährlich 20000 bis 30000 Arbeitsplätzen im Gesamtunternehmen DBP während der nächsten sechs bis acht Jahre (vgl. Handelsblatt v. 20.5.85). 
Der wachstumsträchtige Telekommunikationsbereich der DBP wird Arbeitsplatzvernichtungen dieses Ausmaßes keinesfalls »auffangen « können, denn auch hier zieht die Einführung der neuen ISDN-Fernmeldetechnologien starken Beschäftigungsabbau nach sich (nach Aussagen aus dem Bundespostministerium und einschlägigen Personalbedarfsprognosen der Verwaltung fallen z.B. im Bereich der Fernmeldevermittlungstechnik bei Einführung der Digitaltechnik ein Drittel aller Arbeitsplätze weg). Die datengestützte Rationalisierung der nichttechnischen Fernmeldedienste (z.B. Buchung, Auskunft, Störungsannahme) gefährdet ca. 6500 Arbeitsplätze (vgl. Gewerkschaftliche Praxis 10/81). Kaum absehbar sind heute die Fernwirkungen möglicher Privatisierungsmaßnahmen v.a. im Bereich der Installation und Wartung von Fernmeldeendgeräten (z.Zt. ca. 30000 Beschäftigte; vgl. Tenzer 1985).

Angesichts dieser Breite der Rationalisierungsbetroffenheit unter den rund 54000 staatlichen Lohnarbeitern bei der DBP und aufgrund der durch Beamtenstatus und Rationalisierungsschutztarifverträge bestehenden weitreichenden Beschäftigungsgarantien ist die Postverwaltung zur Durchsetzung ihrer Rationalisierungsmaßnahmen auf eine Vielzahl von Praktiken des »flexiblen « Beschäftigungsabbaus unter Vermeidung von offenen Massenentlassungen angewiesen: Ausnützen »natürlicher« Beschäftigtenabgänge, Ausweitung des ohnehin schon außerordentlich hohen Anteils von Teilzeitbeschäftigung, vermehrte Anwendung von Methoden der »kapazitätsorientierten variablen Arbeitszeit « u.a.m. Bereits heute sind die Personalräte der DPG in vielen Bereichen mit einer Flut von Personalumsetzungen auf betrieblicher, lokaler und regionaler Ebene konfrontiert, die die Grenzen personalrätlicher Rationalisierungsschutzpolitik deutlich werden läßt (das Eintreten der DPG für die 35Stunden Woche in der Tarifrunde 1984 war auch ein Versuch, auf solche Probleme eine Antwort zu finden).

Entscheidend für eine erfolgreiche gewerkschaftliche Stategie der Beschäftigungssicherung wird es sein, ob es gelingt, die einheitliche Unternehmensstruktur der DBP zu erhalten; denn die Kommerzialisierung der DBP nach dem »profit-center« Prinzip zielt auch auf eine Segmentierung und Fragmentierung gewerkschaftlicher Interessenvertretung durch die Aufspaltung des Unternehmens in »ökonomische» und »unökonomische« Betriebseinheiten (wie z.B. in Staatsbetrieben in Großbritannien, etwa bei der Kohlebehörde, offensiv praktiziert). Damit wäre langfristig auch die vom Funktionieren einen einheitlichen, hierarchisierten Systems der gesetzlichen Personalvertretung geprägte Struktur der Betriebsgewerkschaft DPG in Frage gestellt, zumal die Entwicklung einer Beschäftigungssicherungsstrategie auf tarifpolitischer Ebene an die Grenzen der repressiven Einschränkung der Streikmöglichkeiten (und damit letzten Endes der Tariffähigkeit) der DPG durch die höchstrichterliche Rechtssprechung zum Beamtenstreik stößt.

In Anbetracht solcher Perspektiven zeigt sich einmal mehr das Dilemma der sozialdemokratischen Vorstellung von einer »sozialen Steuerung « weltmarktorientierter Modernisierungspolitik (vgl. Glotz 1985, S. 208). Dieses»Modernisierungsdilemma der Gewerkschaften «(vgl. Briefs 1985) ist aber im Falle der Telekommunikation nicht nur ein technologiepolitisches, d.h. das Problem des Akzeptierens oder Nicht-Akzeptierens einer bestimmten Form von kapitalistisch determinierter Technik. Vielmehr ist grundsätzlich die Frage nach der Rolle des Staates in der kapitalistischen Krisenentwicklung "jenseits des Keynesianismus aufgeworfen: auf der einen Seite steht die (keynesianisch geprägte) gewerkschaftliche Forderung nach der »Gemeinwohlorientierung« öffentlicher Dienstleistungen (vgl. z.B. Rittershofer 1977), auf der anderen Seite kapitalseitig unabweisbarer Zwang zur - wenn auch nur schrittweisen - Beteiligung am internationalen »Deregulierungswettlauf in der Telekommunikation. 
Für die DPG ist die Erhaltung des staatlichen Post- und Fernmeldemonopols eine Frage von existenzieller Bedeutung. Auf die Antworten der SPD darf man gespannt sein (Peter Glotz arbeitet sich als Mitglied der Regierungskommission Fernmeldewesen gerade in die Materie ein).

Die DPG hat bezüglich der Rolle des Staates als Träger der nachrichtentechnischen Infrastruktur eindeutige programmatische Grundlagen (vgl. DPG 1974). In der Perspektive des Grundsatzprogramms von 1974 ist durchaus eine gewerkschaftliche Position begründbar, die den Erhalt des Fernmeldemonopols und einer einheitlichen Unternehmensstruktur der DBP mit einer konsequenten Politik der Ausweitung der öffentlichen politischen Kontrolle des Kommunikationswesens und seiner Gestaltung, mit der Forderung nach einer durchgreifenden Verkürzung der Arbeitszeit und einer offensiv praktizierten tariflichen Beschäftigungs- und Qualifikationssicherungspolitik verbindet (zur Technikdiskussion in der DPG vgl. die ausführliche Darstellung von Thöne 1984). Demgegenüber steht aber - oftmals unausgesprochen - die Befürchtung, durch eine allzu harte Kritik an der Technologiepolitik der DBP und den damit verbundenen Rationalisierungsauswirkungen Wasser auf die Mühlen der von konservativer Seite geführten Deregulierungsdiskussion zu leiten (vgl. Wellmann/Sommer 1985).

Tatsache bleibt, daß die weltmarktorientierte Modernisierung der Telekommunikation durch den Ausbau des ISDN-Netzes und die Rationalisierung, Deregulierung und Kommerzialisierung der Bundespost nur die beiden Seiten einer Medaille sind. Die Selbstverpflichtung sozialdemokratischer Modernisierungspolitik auf die ökonomische Sachzwanglogik der Weltmarktkonkurrenz ist damit einmal mehr zur Diskussion gestellt.

\section{Anmerkungen}

1 Solche unterschiedlichen Aussagen über die Rangstellung wichtiger Exportländer auf dem Weltmarkt ergeben sich immer wieder aufgrund der unscharfen statistischen Abgrenzung der Produktgruppe »Ausrüstungsgüter der Telekommunikation«.

2 Unter ISDN(= Integrated Services Digital Network) versteht man technisch die Integration des bestehenden Fernsprechnetzes mit dem Daten- und Fernschreibnetz auf der Basis einer einheitlichen digitalen Vermittlungstechnik. Das Schlagwort »Verkabelung ist in dieser Beziehung insofern irreführend, ja verharmlosend, als die Integration der verschiedenen Kommunikationsnetze bereits auf Grundlage des heute vorhandenen Fernmeldekabelnetzes möglich ist und betrieben wird.

\section{Literatur}

Altvater, E. (1983): „Der Kapitalismus in einer Formkrise. Zum Krisenbegriff in der politischen Ökonomie und ihrer Kritik.« in: Argument-Sonderband 100

ders./Hübner, K./Stanger, M. (1983): »Alternative Wirtschaftspolitik jenseits des Keynesianismus«. Berlin.

Armanski, G./Penth, B./Pohtmann, J. (1976): "Lohnarbeit in öffentichen Dienst der BRD«. Berlin. Arnold, D. (1984): "Die künftige Entwicklung der öffentlichen Fernmeldenetze und ihre Auswirkungen auf die Benutzer«. Hamburg, Scientific Control Systenus GmbH.

ders. (1985): »Umstrittenes Monopol«; in: Wirtschaftswoche v. 5.4.85. 
Batstone, E./Ferner, A./Terry, M. (1984): „Consent and Efficiency: Labour Relations and Management in the State Enterprise Oxford.

BDI, BdA, DIHT u.a. (1986): "Fragen der statlichen Aufgabenstellungr und der Organisation im Fernneldewesen «; Stellungnahmen der Spitzenverbände der Wirtschaft vor der Regierungsknmmission Fernmeldewesen. o.O.

Benz-Overhage, K./Brunlop, E./v. Frevberg, T./Papadinitriou, Z. (1982): Neue Technologien und alternative Arbeitsplatzgestaltung«. Frankfurt/New York.

Bleicher, S. (1985): "Auf optimistischem Kursu; in: Die Zeit v. 20.9.85.

BMFT - Bundesninisterium für Forschung und Technologie (1984): „Konyeption der Bundesregie»rung zur Förderung der Entwicklung der Mikroelektronik, der Informations- und Kommunikationstechniken«. Bonn.

Bock, E. (1985): „Staatliche Technologiepolitik und öffentliche Unternehmen - die ordnungs- und strukiurpolitischen Vorstellungen des DGB«; Referat aus der technologiepolitischen Konfereny des Deutschen Gewerkschaftsbundes, 12.-14.9.1985, Bonn.

Briefs, U. (1985): »Technologiepolitik - aber welche? Das Modernisierungsdilemma der Gewerkschaften«; in: Blätter für deutsche und internationale Politik 12/1985.

CDU (1981): Bundesvorstandsbeschluß Medienpolitik v. 23.7.1981. Bonn.

Deutsche Postgewerkschaft, Bezirk Hessen (1985): „Den Kahlschlagy der gelben Post verhindern«; VLInfo, November 1985.

DIHT - Deutscher Industrie- und Handelstag (1983): »ahresbericht«. Bonn.

Dohmen, A. (1976): „Die Deutsche Bundespost als Auftraggeberin der Elektroindustrie巛; in: Institut für Bilanzanalysen: "Die Elektroindustrie«. Frankfurt.

ders. (1980): „Die Einkaufspolitik der Deutschen Bundespest in Fernmeldewesen «; in: »Zeitschrift für das Post- und Fernmeldewesen « 9/1980.

DPG - Deutsche Postgewerkschaft (1974): „Gesellschafts- und berufspolitisches Programm der Deutschen Postgewerkschaft «. Frankfurt.

Enquête-Kommission Neue Informations- und Kommunikationstechniken des Deutschen Bundestages (1982): »Zwischenbericht «; Drucksache 9/2442 des deutschen Bundestages. Bonn.

Ernst, D. (1984): „Die Auswirkungen der Mikroelektronik auf die weltweite Neust rukturierung der Elektroindustrie - Implikationen für die Dritte Welt«, in: Peripherie 15/16.

Esser, J./Fach, W./Simonis, G. (1980): „Grenzproblente des Modells Deutschland«; in: Prokla 40.

ETUI - European Trade Union Institute (1984): „European Industrial Policy for the Electronics and Information Technology Sector«. Brüssel.

FDP (1984): »22 Thesen zur Fernmelde- und Medienpolitik «. Bronn.

Financial Times (1985): »Teleconmunications: Statistical Trends«; in: Financial Times v. 21. 10.85.

Gizycki, R. v./Schubert, I. (1983): »Microelectronics: A Challenge for Europe's Industrial Survival«. Sozialwissenschaftliche Reihe des Battelle-Instituts Frankfurt, Bd. 9. München.

Glotz, P. (1985): "Arbeit und Technik - Zum Stand der Diskussion«; in: Die Neue Gesellschaft / Frankfurter Hefte 3/1985.

Hauff, V. (1979): »Politik und Elektronik«; Vortrag auf der DPG-Fachtagung Elektronik und Arbeit; in: Gewerkschaftliche Praxis, 4-5/1979.

Hirsch, J. (1980): »Der Sicherheitsstaat . Frankfurt.

Hoppmann, E./Schlögel, H. (1971): »Rationalisierung durch Kartelle - Fernmeldekabelfall«; Schriftenreihe des Vereins für Sozialpolitik, Bd. 62. Berlin.

Ifo-Institut für Wirtschaftsforschung (1983): „Gesamtwirtschaftliche und sektorale Perspektiven der Telekommunikation in der Bundesrepublik Deutschlanda. München.

Junne, G. (1983): »Technologiepolitik und internationale Beziehungen - Der strukturpolitische Wettlauf zwischen den kapitalistischen Industrieländern«; unv. Manuskript. Amsterdam.

Kaufer, E. (1981): »Theorie der öffentlichen Regulierung«. München.

Knieps, G. (1983): »Is Technolugical Revolution a Suffictent Reason for Changing the System of Reyulation? The Case of Telecommunications«; in: v. Weizsäcker, C.C./ Richter, G. (Hg.): »Regulation 
- Analysis and Experience in West Germany and the U.S.«. Zeitschrift für die gesanre Staatswissenschaft $139 / 3$.

Knight-Wendling AG (1985): »Strategisches Konzept für das Postwesen«. Düsseldorf.

Kohl, G. (1982): „Changing Competitive and Technology Environments in Telecommunications ; in: Kennedy, D. e.a.: »Labor and Technology. Unions Response in a Changing Environment火. New York.

Kubicek, H./Rolf, A. (1985): »Mikropolis«. Hamburg.

Kubicek, H. (1986): »Für eine andere Technologiepolitik«; in: Hörfunk, Fernsehen, Film 1-2/19.86.

Lüthje, B. (1985): »Telekomnunikationsindustrie und Strukturwandel - Weltmarktkonkurrenz, industrielle Strukturveränderung und Staatsintervention im Telekomnunikationssektor in der BRD«; Dipl. - Arb., Fachbereich Gesellschaftswissenschaften, Uni Frankfurt/M.

Mayer, W. (1986): „Das Fernneldemonopol verbürgt gar nichts«; in: Deutsche Post v. 20.4.1986.

Memorandum (1983): "Situation und Zukunft der Informationstechnik in der Bundesrepublik Deutschland - Memorandum der Arbeitsgemeinschaft informationstechnische Wirtschaft an die Bundesregierung«; Manuskript, o.O.

Mestmäcker, E.-J. (1980): "Fernmeldemonopol und Nachfragemacht«; in: ders. (H乡.): "Kommunikation ohne Monopole«. Baden - Baden.

Meyer, W. - Deutscher Industrie- und Handelstag (1983): »Thesen zur rechtlich-organisatorischen Gestaltung Neuer Medien aus der Sicht der Wirtschaf«. Referat auf dem Fachkongreß Neue Medien des Instituts für Kommunalwissenschaften der Konrad-Adenauer-Stiftung v. 31.8.-2.9. 1983 in Berlin; Manuskript, St. Augustin.

Monopolkommission (1981): "Die Rolle der Deutschen Bundespost im Fernmeldewesen «, Sonderzutachten Nr. 9. Baden-Baden.

Möhring, D. (1976): "Die deutsche Fernmeldeindustrie - Partner der Deutschen Bundespost «; in: Institut für Bilanzanalysen: "Die Elektroindustrie«. Frankfurt/M.

OECD (183): »Telecommunications - Pressures and Policies for Change«. Paris.

Palloix, C. (1979): Internationalisierung des Produktionssystems und Aufteilung in industrielle und finanzielle Branchen am Beispiel der Stahlindustrie sowie der Elektroindustrie und des Maschinenbaus«; in: Deubner/Rehfeldt/Schlupp/Ziebura (Hg.): »Die Internationalisierung des Kapitals«. Frankfurt/New York.

Plettner, B. (1983): "Japan und die USA sind stark, Europa kann aber mithalten«; in: Handelsblatt v. 29.11.83.

Raabe, M./Stöhr, W. (1974): „Die Post in Griff der Konzerne«. Frankfurt/M.

Rittershofer, W. (1977): »Die Investitionspolitik der DBP - Dilem ma zwischen beschäftigungspolitischen Notwendigkeiten und Verpflichtung zur Eigenwirtschaftlichkeit«; in: WSI-Mitteilungen 6/1977.

Schiller,D. (1982): "Telematics and Government«. Norwood, N.J.

ders. (1985): „Die fortschreitende Privatisierung in der Welt-Telekommunikationsindustrie«; in: Prokop, D. (Hg.): »Medienforschung - Band I, Konzerne, Macher, Kontrolleure«. Frankfurt.

Sohn-Rethel, A. (1979): "Ökonomie und Klassenstruktur des deutschen Faschismus«. Frankfurt.

SPD-Medienkommission (1984): »Medienpolitisches Aktionsprogranm) (Kurzfassung)«; in: MediaPerspektiven 2/1984

SVR-Sachverständigenrat zur Begutachtung der gesantwirtschaftlichen Entwicklung (1985): "Jahresgutachten \&onn

Tenzer, G. (1985): »Die Endyerätepolitik der Deutschen Bundespost «; in: Zeitschrift für das Post- und Fernmeldewesen 10/85.

Thöne, W. (1984): "Die Diskussion in der Deutschen Postgewerkschaft un die Einführung 'Neuer Technologien' als Problem gewerkschaftlicher Pollitik; Diplon-Arbeit, FB Gesellschaft wissenschaften, Univ. Frankfurt/M.

Wellmann, M. / Sommer, M. (1985): „Gespaltene Kommunikationsnetze, eine falsche Strategie«; in: Hörfunk, Fernsehen, Film 12/1985. 
Wiley, R. (1980): »Competition and Deregulation in Telecommunications: The American Experience«; in: Mestmäcker, E.J. (Hg.): »Kommunikation ohne Monopole». Baden-Baden.

Zerdick, A. (1984): "Auswirkungen der Informations- und Kommunikationstechniken auf die Wirtschaftsentwicklung«; Kurzreferat auf der DPG-Fachtagung »Kommunikation und Gesellschaft «, 14./15.6.84 in Bonn; Manuskript.

ZVEI - Zentralverband der Elektrotechnischen Industrie, Fachverband Fernmeldetechnik (1983): "Kommunikationstechnik in der Bundesrepublik Deutschland«. Frankfurt. 